\section{Auf Apoplexe folgen häufig Depressionen}

In einer dänischen Studie wurden Daten von knapp 160.000 Schlaganfallpatienten mit Daten einer Referenzgruppe von über 160.000 Probanden verglichen. Gut 25\% der Schlaganfallpatienten entwickelten in den ersten zwei Jahren nach Studieneintritt eine Depression, verglichen mit 8\% in der Referenzgruppe. Besonders gefährdet waren die Insultpatienten in den ersten drei Monaten nach dem Schlaganfall. Hier lag das Risiko fast neunfach höher als in der Vergleichsgruppe. Im zweiten Jahr der Nachbeobachtung lag es nur noch knapp doppelt so hoch.

Jørgensen TSH et al. JAMA Psychiatry 2016;

73:1032-1040

\section{Cranberry schützt nicht vor HWI}

Bei 92 Pflegeheimbewohnerinnen im mittleren Alter von 86 Jahren, die zweimal täglich Cranberry-Kapseln schluckten (etwa $600 \mathrm{ml}$ Cranberrysaft entsprechend), war ebenso häufig eine Bakteriurie in Kombination mit einer Pyurie nachzuweisen wie bei 93 Frauen, die Placebokapseln erhalten hatten $(29,1$ versus 29,0\%). Auch symptomatische Harnwegsinfekte traten in der Verumgruppe nicht signifikant seltener auf (10 versus 12). Der Anteil von E.-coli-Isolaten in den Urinproben der Verumgruppe hatte sich über die Zeit nicht vermindert.

Juthani-Mehta Met al. JAMA. 2016;316(18):1879-87

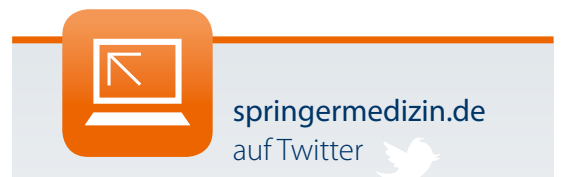

Auch im Herbst heißt es wieder:

Lauschen Sie unserem Zwitschern und werden Sie zum Follower!

twitter.com/springermedizin

Muntermacher kaltes Wasser

Kalt Duschen senkt die

Krankmeldungsrate

Kaltes Duschen soll munter machen und abhärten. $\mathrm{Ob}$ das wirklich stimmt, haben holländische Forscher nun untersucht.

Einem Forscherteam aus Amsterdam gelang es, 3018 Teilnehmer für die erste randomisierte kontrollierte Studie zum Effekt von Kaltduschen auf die Gesundheit zu gewinnen. Die Teilnehmer sollten sich über 30 Tage täglich duschen, und zwar erst nach Belieben warm oder heiß, dann so kalt, wie es aus der Leitung kam.

Die Gesamtgruppe wurde gevierteilt: Ein Viertel musste 30 Sekunden bei $10-12^{\circ} \mathrm{C}$ kaltem Wasser ausharren, ein weiteres 60 Sekunden und die dritte Gruppe 90 Sekunden. Das letzte Viertel diente als Kontrollgruppe; in dieser wurde nach Belieben (warm) geduscht.
Das auffälligste Ergebnis; Die Rate der Krankmeldungen wurde durch die Intervention im Vergleich zur Kontrollgruppe um insgesamt $29 \%$ reduziert. Dabei schien es keine Rolle zu spielen, wie lange man sich den kalten Duschstrahl zugemutet hatte: Die Unterschiede zwischen den Interventionsgruppen in puncto Krankmeldungen waren nicht signifikant.

Was sich nicht zeigen ließ, war ein Effekt auf die Krankheitstage (unabhängig davon, ob man zur Arbeit gegangen war oder nicht). Ob sich die Teilnehmer also nur häufiger zusammengerissen hatten und krank zur Arbeit gegangen waren, bleibt offen.

Buijze GA et al. PLoS One. 2016;11(9):e0161749. Published online 2016 Sep 15. doi: 10.1371/journal.pone.0161749

Testosteron plus Gestagen i.m. für Männer

\title{
Abbruch der Studie zur Verhütungsspritze
}

Durch die Injektion der Kombination aus Testosteron-Undecanoat mit dem Gestagen Norethisteronenanthat (NETE) lässt sich die Spermienbildung wirksam und reversibel unterdrücken. Sie ist allerdings auch mit einigen $\mathrm{Ne}$ benwirkungen verbunden.

Deutsche Forscher haben die kontrazeptive Wirksamkeit durch die Injektion der Kombination aus $200 \mathrm{mg}$ NETE und $1000 \mathrm{mg}$ eines Testosterons i.m. alle acht Wochen bei 320 gesunden Männern im Alter zwischen 18 und 45 Jahren untersucht.

Den Studienergebissen zufolge ließen sich durch die Injektionen Schwangerschaften wirksam verhüten: Nur vier von insgesamt 266 Partnerinnen der behandelten Männer wurden während des Behand- lungszeitraums schwanger (1,57 pro 100 Anwendern). Das entspricht den Ärzten zufolge einem Pearl-Index von 2,18 Schwangerschaften pro 100 Personenjahre. Allerding wurde die im September 2008 begonnene Studie wegen einiger unerwünschter Wirkungen im März 2011 vorzeitig beendet. $29 \%$ der insgesamt fast 1500 unerwünschten Ereignisse wurden als sicher mit der Hormonbehandlung assoziiert bewertet. Die häufigsten Nebenwirkungen waren Akne ( $n=147)$, Schmerzen an der Einstichstelle ( $\mathrm{n}=74)$, Stimmungsschwankungen $(\mathrm{n}=15)$ und Depressionen $(n=9) .122$ Männer gaben an, eine gesteigerte Libido $\mathrm{zu}$ haben.

(ple)

Behre HM et al. J Clin Endocrinol Metab 2016; online 27. Oktober.doi: 10.1210/jc.2016-2141 